Sancho et al. Reply: Upon rechecking our numerical procedure in [1] and its extension [2], we found a programing error that resulted in a different correlation function of the random potential than that given in our Letter. Our numerical results for the random case (but only for the random case) were affected by the error. We note that some mathematical results point to the dependence of superdiffusion in random potentials on the particular form of the force correlations [3]. We have repeated our simulations for the original parameter values, and find, in agreement with the preceding Comment [4], that the system is diffusive for a friction of 0.0001 . However, for frictions of 0.008 and 0.001 the system relaxes to a trapping (rather than a subdiffusive) regime, indicating that the particles do not have sufficient thermal energy to overcome the potential barriers within the simulation observation time.

Our numerical error notwithstanding, we firmly stand by our claim that Brownian particles moving over a surface with a random potential of the type described in our work may be superdiffusive, albeit for other parameter values. Thus, while the Comment is correct about the specific numerical results we presented, the general existence of a superdiffusive parameter regime is still under scrutiny. The occurrence of superdiffusion depends on a few trajectories which may be very sensitive not only to small changes in parameter values but also to numerical algorithms and to the statistics over an ensemble of particles and of realizations of the random potential. Indeed, the whole issue of the range of behaviors (from superdiffusive to subdiffusive) at intermediate times is interesting and largely uncharted territory, and yet the entire range of behaviors seems to appear in experimental studies. For instance, experiments on intracellular transport of therapeutic gene carriers show behaviors from subdiffusive to superdiffusive [5], while gold nanocluster motion on a graphite surface seems to be superdiffusive [6].

We agree about the importance of finite-size limitations. However, since their own results for $L=4096$ (the size of our lattice) and for double this size are essentially identical as far as we can ascertain from their figure, the smaller size seems sufficient for these results. In any case, simulations in larger systems are always desirable.

We have carried out (corrected) simulations of our random system and have found that an increase of the noise intensity (i.e., of the scaled temperature $\mathcal{T}$ ) or a reduction of the mean potential height $\varepsilon$ can lead to anomalous diffusion. A sampling of our results is shown in Fig. 1, displaying superdiffusive, diffusive, and subdiffusive regimes. We conclude that, as claimed in our original Letter, (but for different parameter values) one does observe, in the interval of time of the simulations, the entire gamut of behaviors in the random potential with Gaussian correlations, but agree that the current numerical and experimental evidence needs to be complemented with more extensive numerical work and, if possible, with analytic work.

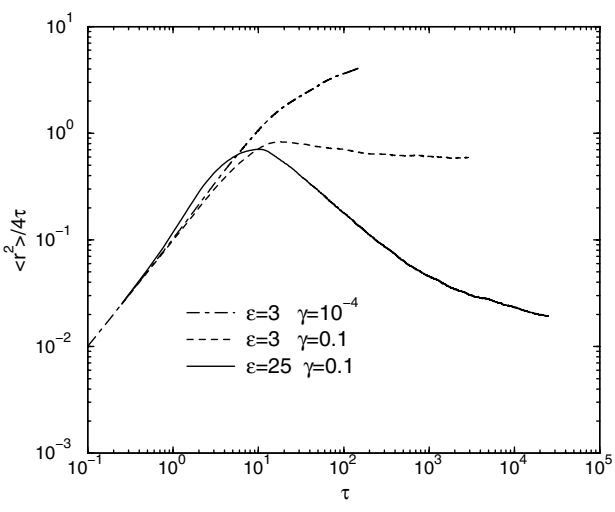

FIG. 1. $\left\langle r^{2}\right\rangle / 4 \tau$ for three sets of values of the random potential intensity parameter $\varepsilon$ and the damping coefficient $\gamma$ as defined in [1]. Other parameters are $N=4000, \mathcal{T}=0.2$, and $\Delta x=0.5$.

\section{J.M. Sancho}

Departament d'Estructura i Constituents de la Matèria

Facultat de Física

Universitat de Barcelona

Diagonal 647

E-08028 Barcelona, Spain

A. M. Lacasta

Departament de Física Aplicada

Universitat Politècnica de Catalunya

Avinguda Dr. Marañon

44 E-08028 Barcelona, Spain

Katja Lindenberg

Department of Chemistry and Biochemistry 0340

University of California

San Diego La Jolla, California 92093-0340, USA

I. M. Sokolov

Institut für Physik

Humboldt Universitæt zu Berlin

Newtonstr. 15, 12489 Berlin, Germany

A. H. Romero

CINVESTAV

Departamento de Materiales

Unidad Querétaro Querétaro, Mexico 76230

Received 18 March 2005; published 12 May 2005

DOI: 10.1103/PhysRevLett.94.188902

PACS numbers: 05.40.-a, 68.35.Fx, 68.43.Jk

[1] J. M. Sancho, A. M. Lacasta, K. Lindenberg, I. M. Sokolov, and A. H. Romero, Phys. Rev. Lett. 92, 250601 (2004).

[2] A. M. Lacasta, J. M. Sancho, A. H. Romero, I. M. Sokolov, and K. Lindenberg, Phys. Rev. E 70, 051104 (2004).

[3] T. Komorowsky and S. Olla, J. Stat. Phys. 108, 647 (2002).

[4] Jing-Dong Bao and Yan Zhou, preceding Comment, Phys. Rev. Lett. 94, 188901 (2005).

[5] J. Suh, D. Wirtz, and J. Hanes, Proc. Natl. Acad. Sci. U.S.A. 100, 3878 (2003).

[6] W. D. Luedtke and U. Landman, Phys. Rev. Lett. 82, 3835 (1999). 